\title{
Phase Transition Model for Traffic at a Junction
}

\author{
Rinaldo M. Colombo \\ Dept. of Mathematics \\ University of Brescia \\ 25133 Brescia \\ Italy
}

\author{
Mauro Garavello \\ Dept. of Mathematics and Applications \\ University of Milano - Bicocca \\ 20125 Milano \\ Italy
}

August 31, 2005

\begin{abstract}
Consider a junction with $n$ incoming and $m$ outgoing roads. Along each road, the flow of traffic is described through the continuum model displaying phase transitions introduced in [5]. This note provides a Riemann solver for the resulting Riemann problem at the junction.
\end{abstract}

2000 Mathematics Subject Classification: 35L65, 90B10.

Key words and phrases: Phase transition model, Riemann problem.

\section{Introduction}

This paper extends the continuum model for traffic introduced in [5] to comprehend junctions. The model is based on conservation laws and allows for the description of phase transitions. The macroscopic variables are the density of vehicles and the mean traffic speed or, equivalently, the density and the weighted momentum. Essentially, below a threshold speed, the flow of traffic is in the congested phase and the model consists of the $2 \times 2$ system of conservation laws introduced in [4]. At higher speeds, the traffic is in the free phase and is satisfactorily described by the classical Lighthill-Whitham and Richards (LWR) model $[9,10]$. The global well posedness of this model is proved in [6], while possible source terms are introduced and evaluated in [2]. We refer to these papers for further details.

In the recent literature, several results were obtained on road or telecommunication networks. We refer in particular to [3], where the LWR model is considered, and to [8] which is related to the Aw-Rascle model [1]. In these papers, junctions are modeled so that the presence of a single fully congested outgoing road immediately saturates the junction and no vehicle may pass through it. On the contrary, here we postulate that the flow through a junction vanishes only in two situations: either all the outgoing roads are fully congested, or the incoming roads are empty. The approach 
used in [7] with reference to telecommunication networks is similar to the one followed here along outgoing roads. However, a typical feature of traffic flows at junction is the presence of precedences. Here, they enter in the definition of the solution to Riemann problem along the roads entering the junction.

Assume $n$ roads enter a junction and $m$ roads exit from it, see Figure 1 . Along each road the traffic density and speed are initially uniformly constant and their evolution is described by the model [5]. We provide below a solution to the resulting Riemann problem. As in $[3,7,8]$, this solution is

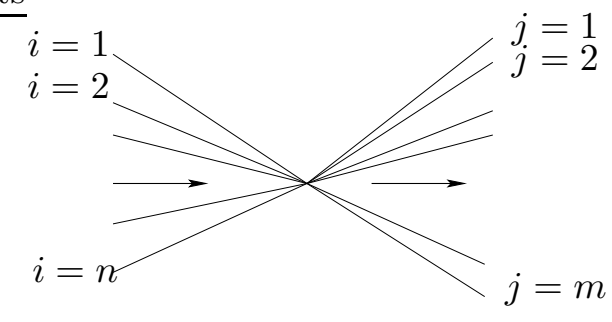

Figure 1: A junction.

a necessary preliminary step to the solution of the general Cauchy problem on a net.

\section{The Basic Model}

We briefly recall here the model [5], which we use later to describe the evolution of the traffic flow along each road. For notational simplicity, in this paragraph we omit the indexes referring to each single road entering the junction.

$$
\begin{array}{ll}
\text { Free flow: }(\rho, q) \in \Omega_{f} & \text { Congested flow: }(\rho, q) \in \Omega_{c} \\
\partial_{t} \rho+\partial_{x}[\rho \cdot v]=0 & \left\{\begin{array}{l}
\partial_{t} \rho+\partial_{x}[\rho \cdot v]=0 \\
\partial_{t} q+\partial_{x}[(q-Q) \cdot v]=0
\end{array}\right. \\
v=\left(1-\frac{\rho}{R}\right) \cdot V & v=\left(1-\frac{\rho}{R}\right) \cdot \frac{q}{\rho} .
\end{array}
$$

Here, $\rho$ is the traffic density, $v$ the average vehicle speed, $q$ the weighted linear momentum, $R$ and $V$ the maximal vehicle density and speed respectively. Finally, from the traffic point of view, the parameter $Q$ is characterized by the phenomenon of wide jams, see [5] for further details. The free and the congested phase, see Figure 2, are respectively defined as

$$
\begin{aligned}
& \Omega_{f}=\left\{(\rho, q) \in[0, R] \times\left[0,+\infty\left[: v_{f}(\rho) \geq V_{f}, q=\rho \cdot V\right\}\right.\right. \\
& \Omega_{c}=\left\{(\rho, q) \in[0, R] \times\left[0,+\infty\left[: v_{c}(\rho, q) \leq V_{c}, \frac{q-Q}{\rho} \in\left[\frac{Q^{-}-Q}{R}, \frac{Q^{+}-Q}{R}\right]\right\} .\right.\right.
\end{aligned}
$$




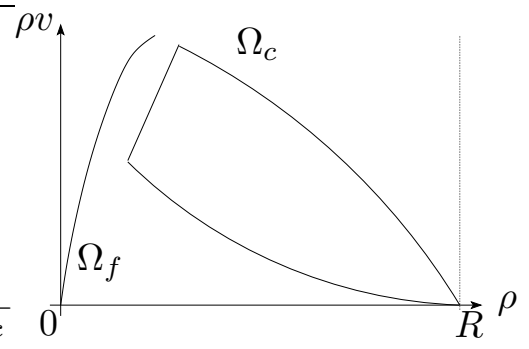

Figure 2: The fundamental diagram for (2.1).

Here, $V_{f}$ and $V_{c}$ are the threshold speeds, i.e. above $V_{f}$ the flow is free, while below $V_{c}$ the flow is congested. Following $[5,6]$, throughout the present note we assume that the various parameters are strictly positive and satisfy

$$
\begin{gathered}
V>V_{f}>V_{c}, \quad \frac{Q^{+}-Q}{R V}<1 \quad \text { and } \quad V_{f}=\frac{V-Q^{+} / R}{1-\left(Q^{+}-Q\right) /(R V)} . \\
Q^{+} \geq Q \geq Q^{-},
\end{gathered}
$$

Finally, we recall the condition

$$
\left(1-\frac{Q^{+}}{R V}\right) \cdot\left(\frac{Q^{+}}{Q}-1\right)<1,
$$

that ensures that all 1-Lax waves have negative speed, see [6, Proposition 2.3].

\section{The Riemann Problem at a Junction}

Consider a crossing with $n$ incoming roads where, say, $x \in]-\infty, 0]$, and $m$ outgoing roads, where $x \in[0,+\infty[$. We assume that the system (2.1) describes the evolution of traffic flow on each incoming road and on each outgoing road. Note that we allow the road parameters to be different among the different roads. Therefore, we refer below to $\Omega_{i}$ or $\Omega^{j}$ for the state space of the $i$-th incoming or $j$-th outgoing road, to $R_{i}$ or $R^{j}$ for the maximal density along the $i$-th incoming or $j$-th outgoing road, and so on.

The interplay of roads at the crossing is modeled through the preferences among the outgoing roads of driver and the order of precedence among the incoming roads.

The strictly positive parameters $\theta^{1}, \ldots, \theta^{m}$, normalized so that $\sum_{j=1}^{m} \theta^{j}=$ 1 , describe the drivers' preferences. More precisely, let $F$ be the total through-flow at the crossing. If the capacity of the $j$-th road allows for it, $\theta^{j} F$ is the flow entering the $j$-th road.

On the other hand, the indexing of the incoming road gives the order of precedence, in the sense that the first vehicles that cross the junction are 
those coming from the first road. If the capacity of the outgoing roads is sufficiently large, then also the vehicles coming from the road 2 may cross the junction, then those coming from road 3, and so on.

By Riemann problem at this junction we mean the Cauchy problem consisting of $n+m$ models $(2.1)$ defined for $(t, x) \in[0,+\infty[\times]-\infty, 0]$ and for $(t, x) \in[0,+\infty[\times[0,+\infty[$, (each with its own parameters), equipped with initial data in the corresponding state space $\Omega_{i}$ or $\Omega^{j}$. More precisely:

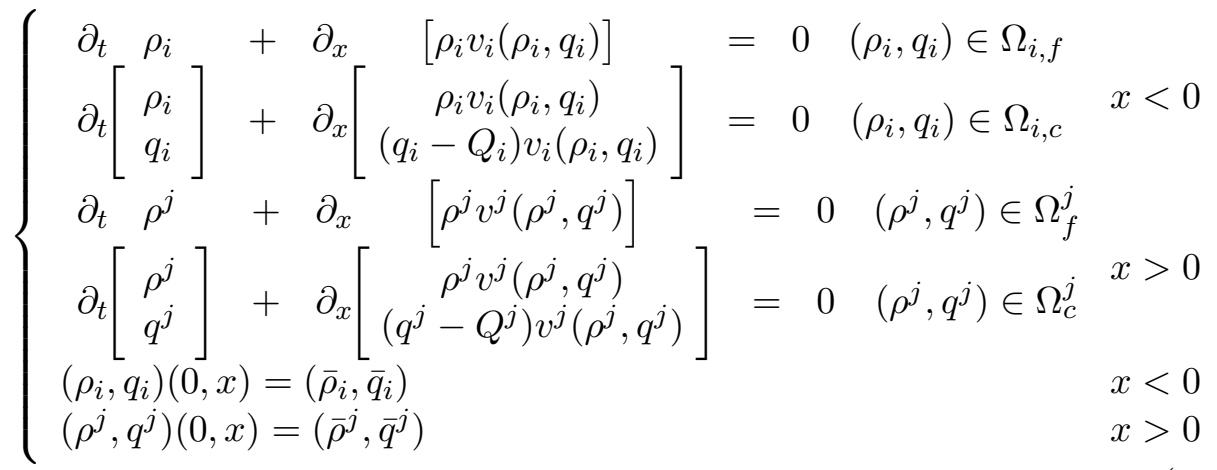

where $\left(\bar{\rho}_{i}, \bar{q}_{i}\right) \in \Omega_{i},\left(\bar{\rho}^{j}, \bar{q}^{j}\right) \in \Omega^{j}$ and we set

$$
v_{i}\left(\rho_{i}, q_{i}\right)= \begin{cases}\left(1-\frac{\rho_{i}}{R}\right) \cdot V_{i} & \text { if }\left(\rho_{i}, q_{i}\right) \in \Omega_{i, f} \\ \left(1-\frac{\rho_{i}}{R}\right) \cdot\left(q_{i} / \rho_{i}\right) & \text { if }\left(\rho_{i}, q_{i}\right) \in \Omega_{i, c} .\end{cases}
$$

$v^{j}$ is defined similarly. A natural space for the solution to Riemann problems consists of $\mathbf{B V}$ perturbation of a given Riemann data, equipped with the $\mathbf{L}^{\mathbf{1}}$ distance. In other words, for any state $\bar{u}=(\bar{\rho}, \bar{q})$ we define

$$
\begin{aligned}
& \left.\left.\left.\left.\mathbf{X}_{i}(\bar{u})=\mathbf{B V}(]-\infty, 0\right] ; \Omega_{i}\right) \cap\left(\bar{u}+\mathbf{L}^{\mathbf{1}}(]-\infty, 0\right] ; \mathbb{R}^{2}\right)\right) \\
& \mathbf{X}^{j}(\bar{u})=\mathbf{B V}\left(\left[0,+\infty\left[; \Omega_{i}\right) \cap\left(\bar{u}+\mathbf{L}^{\mathbf{1}}\left(\left[0,+\infty\left[; \mathbb{R}^{2}\right)\right)\right.\right.\right.\right.
\end{aligned}
$$

Definition 3.1 Fix the end states $\bar{u}_{i} \in \Omega_{i}$ and $\bar{u}^{j} \in \Omega^{j}$ for $i=1, \ldots, n$ and $j=1, \ldots, m$. By weak solution to (3.1), we mean a set of self-similar functions

$$
\begin{aligned}
\left(\rho_{i}, q_{i}\right) & \in \mathbf{C}^{\mathbf{0}}\left(\left[0,+\infty\left[; \mathbf{X}_{i}\left(\bar{u}_{i}\right)\right)\right.\right. \\
\left(\rho^{j}, q^{j}\right) & \in \mathbf{C}^{\mathbf{0}}\left(\left[0,+\infty\left[; \mathbf{X}^{j}\left(\bar{u}^{j}\right)\right)\right.\right.
\end{aligned}
$$

such that

1. $\left(\rho_{i}, q_{i}\right)$ is a weak entropic solution to $(2.1)_{i}$ for $\left.x \in\right]-\infty, 0[$;

2. $\left(\rho^{j}, q^{j}\right)$ is a weak entropic solution to $(2.1)_{j}$ for $\left.x \in\right] 0,+\infty[$; 
3. for all $t \in] 0,+\infty[$,

$$
\lim _{x \rightarrow 0-} \sum_{i=1}^{n} \rho_{i}(t, x) v\left(\rho_{i}(t, x), q_{i}(t, x)\right)=\lim _{x \rightarrow 0+} \sum_{j=1}^{m} \rho^{j}(t, x) v\left(\rho^{j}(t, x), q^{j}(t, x)\right) .
$$

By self-similar we mean that for $i \in\{1, \ldots, n\}$,

$$
\left(\rho_{i}, q_{i}\right)(t, x)=\left(\rho_{i}, q_{i}\right)(\lambda t, \lambda x)
$$

for every $\lambda>0, t>0$ and $x<0$ and for $j \in\{1, \ldots, m\}$,

$$
\left(\rho^{j}, q^{j}\right)(t, x)=\left(\rho^{j}, q^{j}\right)(\lambda t, \lambda x)
$$

for every $\lambda>0, t>0$ and $x>0$.

Note that condition 3 in Definition 3.1 ensures the conservation of vehicles at the junction.

We adapt the standard definition of Riemann solver to the present situation.

Definition 3.2 A Riemann solver for (3.1) is a map $\mathcal{R}$ defined on all states $\prod_{i=1}^{n} \Omega_{i} \times \prod_{j=1}^{m} \Omega^{j} \mapsto \prod_{i=1}^{n} \Omega_{i} \times \prod_{j=1}^{m} \Omega^{j}$ that to any $n$-tuple of pairs $\left(\bar{\rho}_{i}, \bar{q}_{i}\right) \in$ $\Omega_{i}$ and to any m-tuple of pairs $\left(\rho^{j}, q^{j}\right) \in \Omega^{j}$ associates a weak solution to (3.1) computed at time $t=1$.

By Definition 3.1, any weak solution to (3.1) is uniquely determined by its trace at the junction computed at any positive time.

The notion of weak solution at a junction is not sufficient to single out a unique solution to (3.1). Indeed, neither Definition 3.1 nor Definition 3.2 are related to any sort of stability requirement or entropy condition. Consider the case $n=m=1$. Then, (3.1) reduces to a standard Riemann problem and any of its infinitely many weak solutions is a weak solution to (3.1) in the sense of Definition 3.1.

We first state some lemmas about weak solutions to (3.1).

Lemma 3.3 Consider the Riemann problem (3.1) and fix an $i$ in $\{1, \ldots, n\}$. Let $v_{i}=v_{i}\left(\rho_{i}, q_{i}\right)$ as in (3.2). Then, there exist two continuous functions $\alpha_{i}=\alpha_{i}\left(\rho_{i}, q_{i}\right)$ and $\beta_{i}=\beta_{i}\left(\rho_{i}, q_{i}\right)$ such that every weak solution to (3.1) satisfies

$$
\lim _{x \rightarrow 0^{-}} \rho_{i}(1, x) \cdot v_{i}\left(\rho_{i}(1, x), q_{i}(1, x)\right) \in\left[0, \alpha_{i}\left(\rho_{i}, q_{i}\right)\right] \cup\left\{\beta_{i}\left(\rho_{i}, q_{i}\right)\right\} .
$$

Moreover, for every $\eta \in\left[0, \alpha_{i}\left(\rho_{i}, q_{i}\right)\right] \cup\left\{\beta_{i}\left(\rho_{i}, q_{i}\right)\right\}$, there exists a weak solution to the Riemann problem (3.1) such that

$$
\lim _{x \rightarrow 0^{-}} \rho_{i}(1, x) \cdot v\left(\rho_{i}(1, x), q_{i}(1, x)\right)=\eta .
$$


The proof is postponed to Section 4, where the explicit expressions of $\alpha_{i}$ and $\beta_{i}$ are provided. The analogous statement for outgoing roads is the following.

Lemma 3.4 Consider the Riemann problem (3.1) and fix a $j \in\{1, \ldots, m\}$. Then, there exists a continuous function $\gamma^{j}=\gamma^{j}\left(\rho^{j}, q^{j}\right)$ such that every weak solution to (3.1) satisfies

$$
\lim _{x \rightarrow 0^{+}} \rho^{j}(1, x) \cdot v^{j}\left(\rho^{j}(1, x), q^{j}(1, x)\right) \in\left[0, \gamma^{j}\left(\rho^{j}, q^{j}\right)\right] .
$$

Moreover, for every $\eta \in\left[0, \gamma^{j}\left(\rho^{j}, q^{j}\right)\right]$, there exists a weak solution to the Riemann problem (3.1) such that

$$
\lim _{x \rightarrow 0^{-}} \rho_{i}(1, x) \cdot v\left(\rho_{i}(1, x), q_{i}(1, x)\right)=\eta .
$$

For $i=1, \ldots, n$ and $j=1, \ldots, m$ define the sets

$$
\begin{aligned}
\mathcal{F}_{i}\left(\rho_{i}, q_{i}\right) & =\left[0, \alpha_{i}\left(\rho_{i}, q_{i}\right)\right] \cup\left\{\beta_{i}\left(\rho_{i}, q_{i}\right)\right\} \\
\mathcal{F}^{j}\left(\rho_{i}, q_{i}\right) & =\left[0, \gamma^{j}\left(\rho^{j}, q^{j}\right)\right] .
\end{aligned}
$$

Let

$$
\Delta_{\text {out }}\left(\varphi^{1}, \ldots, \varphi^{m}\right)=\inf _{\lambda \in[0,+\infty[}\left\|\left(\varphi^{1}, \ldots, \varphi^{m}\right)-\lambda\left(\theta^{1}, \ldots, \theta^{m}\right)\right\|,
$$

where $\|\cdot\|$ stands for the usual Euclidean norm. To select among the infinitely many weak solutions, we adopt the following constructive procedure.

1. The through-flow at the crossing is

$$
F=\max \left(\left(\sum_{i=1}^{n} \mathcal{F}_{i}\left(\bar{\rho}_{i}, \bar{q}_{i}\right)\right) \cap\left(\sum_{j=1}^{m} \mathcal{F}^{j}\left(\bar{\rho}^{j}, \bar{q}^{j}\right)\right)\right)
$$

here the sum between sets is intended in the sense $A+B=\{a+b: a \in$ $A$ and $b \in B\}$. Note that this set is not empty, since it contains at least $\{0\}$.

2. Define recursively the incoming flows respecting the precedences as follows:

$$
\begin{aligned}
\tilde{f}_{1} & =\max \left(\mathcal{F}_{1}\left(\bar{\rho}_{1}, \bar{q}_{1}\right) \cap[0, F]\right) \\
\tilde{f}_{i} & =\max \left(\mathcal{F}_{i}\left(\bar{\rho}_{i}, \bar{q}_{i}\right) \cap\left[0, F-\sum_{l=1}^{i-1} \tilde{f}_{l}\right]\right) \quad i=2, \ldots, n .
\end{aligned}
$$


3. Lemma 3.4 ensures the convexity of the set

$$
J=\left\{\left(\varphi^{1}, \ldots, \varphi^{m}\right) \in \prod_{j=1}^{m}\left[0, \gamma^{j}\left(\bar{\rho}^{j}, \bar{q}^{j}\right)\right]: \sum_{j=1}^{m} \varphi^{j}=F\right\}
$$

so that the outflows

$$
\left(\tilde{f}^{1}, \ldots, \tilde{f}^{m}\right)=\underset{\left(\varphi^{1}, \ldots, \varphi^{m}\right) \in J}{\operatorname{argmin}} \Delta_{\text {out }}\left(\varphi^{1}, \ldots, \varphi^{m}\right)
$$

are uniquely defined.

4. The traces of the solution at the junction are uniquely defined by

$$
\left\{\begin{array} { l } 
{ \tilde { \rho } _ { i } \cdot v _ { i } ( \tilde { \rho } _ { i } , \tilde { q } _ { i } ) = \tilde { f } _ { i } } \\
{ L _ { 2 } ( \overline { \rho } _ { i } ; \tilde { \rho } _ { i } , \tilde { q } _ { i } ) = \overline { q } _ { i } }
\end{array} \quad \left\{\begin{array}{l}
\tilde{\rho}^{j} \cdot v^{j}\left(\tilde{\rho}^{j}, \tilde{q}^{j}\right)=\tilde{f}^{j} \\
L_{2}\left(\bar{\rho}^{j} ; \tilde{\rho}^{j}, \tilde{q}^{j}\right)=\bar{q}^{j}
\end{array}\right.\right.
$$

5. The solutions along each road are defined solving the standard Riemann problems consisting of $(2.1)_{i}$ along incoming roads, respectively $(2.1)_{j}$ along outgoing roads, and initial data

$$
\begin{aligned}
& \left(\rho_{i}, q_{i}\right)(t, 0)=\left\{\begin{array}{l}
\left(\bar{\rho}_{i}, \bar{q}_{i}\right) x<0 \\
\left(\tilde{\rho}_{i}, \tilde{q}_{i}\right) x>0
\end{array},\right. \text { respectively } \\
& \left(\rho^{j}, q^{j}\right)(t, 0)=\left\{\begin{array}{l}
\left(\tilde{\rho}^{j}, \tilde{q}^{j}\right) x<0 \\
\left(\bar{\rho}^{j}, \bar{q}^{j}\right) x>0 .
\end{array}\right.
\end{aligned}
$$

The next proposition describes the properties of this Riemann solver.

Proposition 3.5 The Riemann Solver $\mathcal{R}$ has the following two properties:

1. if $\left(\bar{\rho}_{i_{*}}, \bar{q}_{i_{*}}\right)=(0,0), i_{*} \in\{1, \ldots, n\}$, then $\mathcal{R}$ provides the same solution in the case the $i_{*}$ road does not exist.

2. if $\bar{\rho}^{j_{*}}=R, j_{*} \in\{1, \ldots, m\}$, then $\mathcal{R}$ provides the same solution in the case the $j_{*}$ road does not exist.

In particular, the flow across the junction may well be non zero even if some of the outgoing roads are fully congested.

Proof. Note that if $\left(\bar{\rho}_{i_{*}}, \bar{q}_{i *}\right)=(0,0)$, then $\alpha_{i_{*}}\left(\bar{\rho}_{i_{*}}, \bar{q}_{i *}\right)=\beta\left(\bar{\rho}_{i_{*}}, \bar{q}_{i_{*}}\right)=0$, hence $\mathcal{F}_{i_{*}}\left(\bar{\rho}_{i_{*}}, \bar{q}_{i *}\right)=\{0\}$, so that

$$
\sum_{i=1}^{n} \mathcal{F}_{i}\left(\bar{\rho}_{i}, \bar{q}_{i}\right)=\sum_{i=1, \ldots, n ; i \neq i_{*}} \mathcal{F}_{i}\left(\bar{\rho}_{i}, \bar{q}_{i}\right)
$$


This shows that the through-flow $F$ does not change if the $i_{*}$ road is suppressed.

Similarly, if $\bar{\rho}^{j_{*}}=R$, then $\gamma^{j_{*}}\left(\bar{\rho}^{j}, \bar{q}^{j}\right)=0$, hence $\mathcal{F}^{j}\left(\bar{\rho}^{j}, \bar{q}^{j}\right)=\{0\}$ and

$$
\sum_{j=1}^{m} \mathcal{F}^{j}\left(\bar{\rho}_{j}, \bar{q}_{j}\right)=\sum_{j=1, \ldots, m ; j \neq j_{*}} \mathcal{F}^{j}\left(\bar{\rho}^{j}, \bar{q}^{j}\right),
$$

showing that $\tilde{f}^{j}=0$, completing the proof.

Remark 3.6 In the case $n=m=1$, the Riemann solver $\mathcal{R}_{\left(\theta_{1}, \ldots, \theta_{n}\right)}^{\left(\theta^{1}, \ldots, \theta^{m}\right)}$ is not the standard Lax solver. In fact, given a junction $J$ with one incoming road $I_{1}$ and one outgoing road $I^{1}$, consider the Riemann problem with the initial data $\left(\bar{\rho}_{1}, \bar{q}_{1}\right) \in \Omega_{f}$ and $\left(\bar{\rho}^{1}, \bar{q}^{1}\right) \in \Omega_{c}$. Assume that

1. $\bar{\rho}_{1} v\left(\bar{\rho}_{1}, \bar{q}_{1}\right)<\bar{\rho}^{1} v\left(\bar{\rho}^{1}, \bar{q}^{1}\right)$;

2. $\frac{V q_{*} R}{R V-Q_{1}+q_{*}} \frac{R V-Q_{1}}{R V-Q_{1}+q_{*}}<\bar{\rho}_{1} v\left(\bar{\rho}_{1}, \bar{q}_{1}\right)$.

In this case the previous procedure gives that $\left(\hat{\rho}_{1}, \hat{q}_{1}\right)=\left(\bar{\rho}_{1}, \bar{q}_{1}\right)$, while the standard Lax solution is given by a wave of the first family with negative speed and by a wave of the second family with positive speed.

\section{Technical Proofs}

Proof of Lemma 3.3. The index $i$ is fixed throughout this proof, hence it is omitted. Refer to Figure 2 and follow the 1-Lax curve exiting $(\rho, q)$. Along this curve, the flow $\rho v(\rho, q)$ attains all values in $[0, \alpha(\rho, q)] \cup\{\beta(\rho, q)\}$ where

$$
\begin{array}{ll}
\alpha(\rho, q)= \begin{cases}\rho \cdot v(\rho, q) & \text { if }(\rho, q) \in \Omega_{f} \text { and } \rho v \leq \varphi \\
\left(1-\frac{R V_{c}}{Q+(q-Q)(R / \rho)}\right) R V_{c} & \text { otherwise; }\end{cases} \\
\beta(\rho, q)= \begin{cases}\rho \cdot v(\rho, q) & \text { if }(\rho, q) \in \Omega_{f} \text { and } \rho v \leq \varphi \\
\frac{Q}{V+(Q-q) / \rho}\left(1-\frac{Q / R}{V+(Q-q) / \rho}\right) & \text { otherwise. }\end{cases}
\end{array}
$$

On the other hand, fix an $\eta \in[0, \alpha(\rho, q)] \cup\{\beta(\rho, q)\}$. Choose null initial data in all the incoming roads but in the $i$-th one, where $\rho_{i}, q_{i}$ are assigned. In one of the outgoing roads, select an initial datum with flow $\eta$ and let all the other outgoing roads, if present, be empty. The obvious weak solution to the resulting Riemann problem has trace $\eta$ at the junction. 

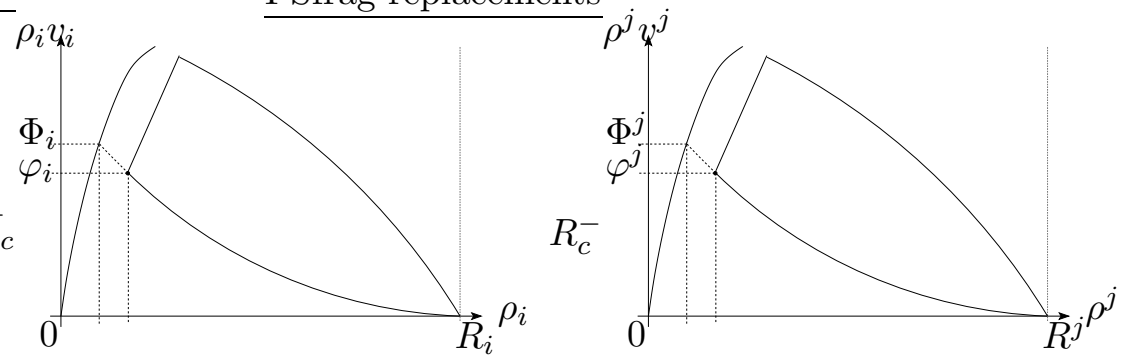

Figure 3: Notation used in Lemma 3.3

Proof of Lemma 3.4. Proceed as in the proof of Lemma 3.3. If $(\rho, q) \in \Omega_{f}$, then the standard Riemann problem with any other point of $\Omega_{f}$ as left state and $(\rho, q)$ as left state is solved by a wave of positive speed. Similarly, assume that $\rho, q) \in \Omega_{c}$. Then, any point lying on its 2-Lax curve as well as any point in $\Omega_{f}$ wit sufficiently small flow have the same property. Along all these points, the flow attains the values $\left[0, \gamma\left(\rho^{j}, q^{j}\right)\right]$, where

$\gamma(\rho, q)= \begin{cases}\left(V-V_{f}\right) \cdot R & \text { if }(\rho, q) \in \Omega_{f} \\ \frac{1}{2} \frac{R}{Q^{+}-Q}\left(Q^{+}-2 Q+K+\sqrt{\left(K+Q^{+}\right)^{2}+4 K Q}\right) & \text { if }(\rho, q) \in \Omega_{c}\end{cases}$

where we set $K=\left(\frac{R}{\rho}-1\right) q$.

\section{References}

[1] A. Aw and M. Rascle. Resurrection of "second order" models of traffic flow. SIAM J. Appl. Math., 60(3):916-938 (electronic), 2000.

[2] P. Bagnerini, R. Colombo, and A. Corli. On the role of source terms in continuum traffic flow models. Technical report, Brescia University, 2005.

[3] G. M. Coclite, M. Garavello, and B. Piccoli. Traffic flow on a road network. SIAM J. Math. Anal., 36(6):1862-1886, 2005.

[4] R. M. Colombo. A $2 \times 2$ hyperbolic traffic flow model. Math. Comput. Modelling, 35(5-6):683-688, 2002. Traffic flow-modelling and simulation.

[5] R. M. Colombo. Hyperbolic phase transitions in traffic flow. SIAM J. Appl. Math., 63(2):708-721, 2002.

[6] R. M. Colombo, P. Goatin, and F. S. Priuli. Global well posedness of a traffic flow model with phase transitions. Technical Report 5, Laboratoire d'Analyse Non Lineaire Appliquee et Modelisation, 2005.

[7] C. D'Apice, R. Manzo, and B. Piccoli. Packets flow on telecommunication networks. Technical report, IAC, Consiglio Nazionale delle Ricerche, Rome, 2005 . 
[8] M. Garavello and B. Piccoli. Traffic flow on a roads network using the aw-rascle model. Comm. Partial Differential Equations.

[9] M. J. Lighthill and G. B. Whitham. On kinematic waves. II. A theory of traffic flow on long crowded roads. Proc. Roy. Soc. London. Ser. A., 229:317-345, 1955 .

[10] P. I. Richards. Shock waves on the highway. Operations Res., 4:42-51, 1956. 